\title{
Cognitive Network Science: A New Frontier
}

\author{
Yoed N. Kenett $\mathbb{D}^{1},{ }^{1}$ Nicole M. Beckage $\mathbb{D}^{2},{ }^{2}$ Cynthia S. Q. Siew $\mathbb{D}^{3},{ }^{3}$ and Dirk U. Wulff $\mathbb{D}^{4,5}$ \\ ${ }^{1}$ Department of Psychology, University of Pennsylvania, Philadelphia, USA \\ ${ }^{2}$ Intel Labs, Hillsboro, USA \\ ${ }^{3}$ National University of Singapore, Singapore \\ ${ }^{4}$ Center for Cognitive and Decision Science, University of Basel, Basel, Switzerland \\ ${ }^{5}$ Max Planck Institute for Human Development, Berlin, Germany
}

Correspondence should be addressed to Yoed N. Kenett; yoedk@sas.upenn.edu

Received 8 January 2020; Accepted 9 January 2020; Published 28 April 2020

Copyright $\odot 2020$ Yoed N. Kenett et al. This is an open access article distributed under the Creative Commons Attribution License, which permits unrestricted use, distribution, and reproduction in any medium, provided the original work is properly cited.

A major challenge in studying the complexity of cognition relates to quantifying abstract theoretical cognitive constructs, such as language, memory, or thinking, and studing the representation of these abstract constructs. Such quantifications of these abstract constructs are based on indirect measures of cognitive systems such as behavioral measures or neural activity. In the past two decades, an increasing number of studies have used network science methods to study complex systems.

Network science is based on mathematical graph theory and offers quantitative methods to investigate complex systems [1]. A network is made up of nodes, which represent the basic unit of the system (e.g., concepts in semantic memory) and links, or edges, which signify the relations between them (e.g., semantic similarity). While the application of network science methodologies has become an extremely popular approach to study brain structure and function [2], it has been used to study cognitive phenomena to a much lesser extent, despite classic cognitive theory in language and memory being highly related to a network perspective [3].

So far, the application of network science to cognitive science has enabled the direct examination of the theory that highly creative individuals have a more flexible semantic memory structure [4], identified mechanisms of language development through network growth modeling [5], shed novel light on statistical learning [6], examined phonological and orthographic effects $[7,8]$, provided new insight into the structure of semantic network of second language in bilinguals [9], and studied changes in memory structure across the lifespan [10].
The aim of this special issue is to demonstrate the potential and strength of applying network science methods to study cognition (broadly defined). In the article "Cognitive Network Science: A Review of Research on Cognition through the Lens of Network Representations, Processes, and Dynamics," C. S. Q. Siew et al. provide a comprehensive review on the field of Cognitive Network Science. Specifically, their article is focused on three key main theses: (1) Network science provides a quantitative approach to represent cognitive systems; (2) network science facilitates a deeper understanding of human cognition by allowing the researcher to consider how network structure and the processes operating on the network structure interact to produce behavioral phenomena; and (3) network science provides a framework to model structural changes in cognitive systems at multiple scales.

It is striking that, without any prior arrangement, the collection of articles in this special issue has aligned rather well with the main theses of the comprehensive review by $\mathrm{C}$. S. Q. Siew et al. Articles by M. Stella, K. D. Neergaard, and C.-R. Huang, S. Letina et al., R. H. Baayen et al., S. M. Herzog and T. T. Hills, A. Mehler et al., and C. Vrijen et al. illustrate how network science methods can be used to represent a variety of cognitive, linguistic, psychological, and even social systems. Articles by M. Stella, K. D. Neergaard and C.-R. Huang, R. H. Baayen et al., S. M. Herzog and T. T. Hills, C. Vrijen et al., and E. A. Karuza et al. demonstrate how the structure of the cognitive network plays an important role in predicting behavioral outcomes in domains including language comprehension and production, statistical learning, 
mental health, and conflict resolution. Finally, articles by $\mathrm{N}$. M. Beckage and E. Colunga and J. C. Zemla and J. L. Austerweil focus on modeling structural changes in the network representation as children learn new words and as cognitive decline sets in.

Furthermore, as apparent from the article summaries below, the collection of articles in this special issue shows how network science approaches can be flexibly applied to address a broad range of topics and domains in the cognitive and social sciences, as well as how network science approaches can creatively advance methodology in these areas. Articles by M. Stella, K. D. Neergaard and C.-R. Huang, A. Mehler et al., and R. H. Baayen et al. show how various aspects of the mental lexicon can be represented as a cognitive network. Articles by E. A. Karuza et al. and N. M. Beckage and E. Colunga focus on how humans learn temporal, event-based visual information, and language, respectively. Articles by S. Letina et al. and C. Vrijen et al. analyzed psychological networks of personality attributes and affect dynamics. Finally, other articles focused on the social graphs of mediators (S. M. Herzog and T. T. Hills), modeling of cognitive decline (J. C. Zemla and J. L. Austerweil), and network methodology (A. Mehler et al. and S. Letina et al.).

Taken together, the articles in this special issue demonstrate the feasibility, and strength, of applying the quantitative language of network science to advance our understanding of complex cognitive phenomena. We present a brief overview of each of the articles in this special issue, according to the order in which they were published.

In the article "Cohort and Rhyme Priming Emerge from the Multiplex Network Structure of the Mental Lexicon," M. Stella used a multiplex lexical network representing both semantic and phonological relationships among words in the mental lexicon to examine two aspects of phonological priming: cohort priming and rhyme priming. Results indicated that both cohort words (i.e., words that share the same initial sounds) and rhyme words (i.e., words that rhyme) were "closer" in terms of distance computed on various layers in the multiplex as compared to random expectation. These results suggest an alternative account of priming effects in psycholinguistics, whereby facilitatory priming may simply emerge as a consequence of higherorder structural relationships among words.

In the article "Spread the Joy: How High and Low Bias for Happy Facial Emotions Translate into Different Daily Life Affect Dynamics," C. Vrijen et al. examined how daily life affect dynamics differed among individuals with low and high levels of bias toward happy facial emotions. Daily-life affect networks refer to networks that represent different emotions (positive/negative) and the effect from one time interval of six hours to the next on these emotions. Specifically, the aim of this study was to examine the importance of laboratory measurement of happy bias in peoples' daily life. Combining a network psychometric approach with experience sampling methodology, the authors found that individuals with high happy bias showed more sustained effects of positive, rewarding experiences in their affect networks over time as compared to individuals with low happy bias. These results suggest that sensitivity to positive experiences may be related to a bias for happy emotions and may act as a buffer against the development of depression.

In the article "The Discriminative Lexicon: A Unified Computational Model for the Lexicon and Lexical Processing in Comprehension and Production Grounded not in (De) Composition but in Linear Discriminative Learning," R. H. Baayen et al. rely on a neural network, trained sentence-bysentence, to predict the occurrence of lexemes. This trained model predicts a variety of behaviors including paired associate learning and semantic relatedness ratings. Moreover, when combined with a phonological representation that maps phonemes to words, they found that the resulting representation allowed them to account for the behaviors recruiting the entire pipeline of visual and auditory comprehension, from word form to meaning. The article suggests methods and applications for learned network representations and how those representations may offer cognitive insight and predict behavior in linguistic experiments.

In the article "Human Sensitivity to Community Structure Is Robust to Topological Variation", E. A. Karuza et al. replicate and extend their previous work by examining how the topology of the environment facilitates statistical learning. E. A. Karuza et al. previous work showed learner sensitivity to the presence of community structure within temporal sequences. However, whether such a sensitivity generalizes to variations in graph topology was unknown. To address this, the authors systematically vary the number and size of communities and assess how it impacts learning. The authors show that learners are sensitive to community structure across a range of network topologies (that vary in their number and size of communities). Thus, this work demonstrates how network science methods can be used to study how individuals are sensitive to the topology of their environment.

In the article "Expanding Network Analysis Tools to Psychological Networks: Minimal Spanning Trees, Participation Coefficients, and Motif Analysis Applied to a Network of 26 Psychological Attributes," S. Letina et al. turn to minimum spanning trees and motif analysis to studying the emerging hierarchy of psychological trait networks. The authors derive a network based on a variety of psychological concepts (correlations of self-reported personality traits from questionnaires such as the Schwatz Value Survey, the Big Five personality traits, Sensational Interest Questionnaire, and others). From this weighted network, they define a minimum spanning tree, participation coefficient, and observed motifs in the original network to study the relationship between these measures and psychological constructs. The authors show how these three types of network analysis, not currently used to study psychological constructs, provides meaningful information and complement each other in the ability to capture and explain the interaction of psychological traits. The authors conclude that certain traits, such as empathy, are central to the network and other nodes, such as intelligence, which are in the periphery still are related to a large number of other traits.

In the article "Constructing the Mandarin Phonological Network: Novel Syllable Inventory Used to Identify Schematic Segmentation", K. D. Neergaard and C.-R. Huang used network science methods to construct various types of 
phonological networks of Mandarin Chinese. These phonological networks were constructed based on various phonological annotation strategies, inferred from a Chinese phonological association task. In this phonological association task, participants produced Chinese syllables that sounded similar to a target Chinese syllable. The authors then use RT data from the phonological association task to identify the optimal annotation strategy to construct the Chinese phonological network. The results indicated that structural aspects of the Chinese phonological network influenced how people "search" for similar sound neighbors in the phonological lexicon. Thus, the authors present a method to systematically study phonological segmentation of languages and how network science can be used to examine how the structure of such an optimally segmented phonological system influence "search processes" operating over it.

In the article "Mediation Centrality in Adversarial Policy Networks," S. M. Herzog and T. T. Hills introduce and explore a new network measure - mediation centrality, a network measure for identifying mediators in bipartite adversarial networks. Adversarial systems can be defined as systems composed of individuals with opposing views, such as Democrats versus Republicans in US politics. Adversarial networks can be represented by bipartite networks, where individuals are connected by edges to the views they support. Over such a bipartite network, a good mediator is an individual (node) that can minimize the polarity of such opposing views. Thus, mediation centrality is computed by combining centrality metrics from subgraph projections where the projections are defined in relation to different sets of views. The authors argue that this measure is important in identifying mediators who can advance conflict resolution in polarized adversarial systems. Finally, S. M. Herzog and T. T. Hills demonstrate the utility of computing mediation centrality across a range of examples, demonstrating its fruitfulness in adversarial systems.

In the article "Analyzing Knowledge Retrieval Impairments Associated with Alzheimer's Disease Using Network Analysis," J. C. Zemla and J. L. Austerweil employ a sophisticated, Bayesian approach to infer an individual's semantic network from just a few number of verbal fluency sequences. The approach is elegant as it is based on a complete cognitive model, encompassing a search process retrieving from an underlying, to-be-inferred representation. Using their modeling approach, J. C. Zemla and J. L. Austerweil are able to generate novel, actionable insights concerning the cognitive development of patients with Alzheimer's disease. Specifically, they show the semantic networks of patients with Alzheimer's disease are less connected, more disordered, and, generally, less smallworld-like than those of healthy controls.

In the article "Network Growth Modeling to Capture Individual Lexical Learning," N. M. Beckage and E. Colunga introduce a network growth modeling framework for quantifying the influence of (1) different network representations, (2) growth processes, and (3) node importance. They test their network growth framework on the prediction of individual language learning trajectories. Their models provide quantification on the emergent structure of young toddler's vocabularies and provide a set of tools to study individual differences in language acquisition trajectories. They show evidence that the acquisition model is influenced by the underlying network representation, the assumed growth process, and the network centrality measure used to quantify the importance of words, highlighting the complex and multifaceted nature of early acquisition. Their framework also provides new tools of analysis and suggests new hypotheses that can be tested in experimental interventions in language development and are targeted at the level of the individual child's current knowledge.

In the article "From Topic Networks to Distributed Cognitive Maps Zipfian Topic Universes in the Area of Volunteered Geographic Information," A. Mehler et al. introduce a set of novel methods to extend the standard analysis of co-occurrence networks from textual corpora based on a multiplex network approach. Specifically, the authors define an approach that allows for thematic comparison directly between different communities by deriving a network of topics from varied sources of information, such as different readership, different authorship, and different medium. The resulting framework introduces a process for deriving such network layers as (1) author topic networks in which connected authors tend to refer to similar thematic elements throughout their writing, (2) text networks which capture the relationship of a single document with other text documents, (3) constituent layers which can be defined to capture such relationships as lexicographic and phrasal information, and (4) contextual layers which link topics based on such high-level features as media and genre. This multiplex topic network approach could allow for modeling of social and cognitive interactions from text-based information sources such as those found on the world wide web.

\section{Conflicts of Interest}

Yoed Kenett declares that he has worked in the past with Dr. Elisabeth Karuza. Nicole Beckage and Yoed Kenett declare that they have worked in the past with Dr. Joseph Austerweil. Dirk Wulff, Cynthia Siew, and Nicole Beckage declare that they have worked in the past with Dr. Thomas Hills. Each editor did not handle work by the named individual they have previously worked with.

Yoed N. Kenett Nicole M. Beckage Cynthia S. Q. Siew

Dirk U. Wulff

\section{References}

[1] A. Baronchelli, R. Ferrer-i-Cancho, R. Pastor-Satorras, N. Chater, and M. H. Christiansen, "Networks in cognitive science," Trends in Cognitive Sciences, vol. 17, no. 7, pp. 348-360, 2013.

[2] J. D. Medaglia, M.-E. Lynall, and D. S. Bassett, "Cognitive network neuroscience," Journal of Cognitive Neuroscience, vol. 27, no. 8, pp. 1471-1491, 2015. 
[3] A. M. Collins and E. F. Loftus, "A spreading-activation theory of semantic processing," Psychological Review, vol. 82, no. 6, pp. 407-428, 1975.

[4] Y. N. Kenett and M. Faust, "A semantic network cartography of the creative mind," Trends in Cognitive Sciences, vol. 23, no. 4, pp. 271-274, 2019.

[5] N. Beckage, L. Smith, and T. T. Hills, "Small worlds and semantic network growth in typical and late talkers," PLoS One, vol. 6, no. 5, Article ID e19348, 2011.

[6] E. A. Karuza, S. L. Thompson-Schill, and D. S. Bassett, "Local patterns to global architectures: influences of network topology on human learning," Trends in Cognitive Sciences, vol. 20, no. 8, pp. 629-640, 2016.

[7] C. S. Q. Siew, "Community structure in the phonological network," Frontiers in Psychology, vol. 4, p. 553, 2013.

[8] C. S. Q. Siew, "The orthographic similarity structure of English words: insights from network science," Applied Network Science, vol. 3, no. 1, p. 13, 2018.

[9] K. Borodkin, Y. N. Kenett, M. Faust, and N. Mashal, "When pumpkin is closer to onion than to squash: the structure of the second language lexicon," Cognition, vol. 156, pp. 60-70, 2016.

[10] D. U. Wulff, S. De Deyne, M. N. Jones, R. Mata, and T. A. L. Consortium, "New perspectives on the aging lexicon," Trends in Cognitive Sciences, vol. 23, no. 8, pp. 686-698, 2019. 